\title{
A case report: robot-assisted resection of a mediastinal Müllerian cyst in a young woman
}

\author{
Yoshikane Yamauchi $^{1} \wedge$, Yuichi Saito ${ }^{1} \wedge$, Koji Saito ${ }^{2}$, Momoko Asami $^{1}$, Toshihiro Haga ${ }^{2}$, Yuko Sasajima ${ }^{2}$, \\ Yasuyuki Kanamoto ${ }^{1}$, Fumi Yokote ${ }^{1}$, Hitoshi Dejima ${ }^{1}$, Yukinori Sakao ${ }^{1}{ }^{\wedge}$, Masafumi Kawamura $^{1}$ \\ ${ }^{1}$ Department of Surgery, Teikyo University School of Medicine, Tokyo, Japan; ${ }^{2}$ Department of Pathology, Teikyo University Hospital, Tokyo, Japan \\ Correspondence to: Yoshikane Yamauchi, MD, PhD. Department of Surgery, Teikyo University School of Medicine, 2-11-1 Kaga, Itabashi-ku, Tokyo \\ 173-8605, Japan. Email: yoshikaney@med.teikyo-u.ac.jp.
}

\begin{abstract}
Mediastinal Müllerian cysts are rare and are composed of ciliated Müllerian-type tubal epithelium. Preoperative diagnosis of this cyst is usually difficult owing to the local anatomy. We report a case of a Müllerian cyst in the mediastinum of a 25-year-old female patient that was resected by robotassisted thoracoscopic surgery (RATS) without rupturing the cyst. The tumor was a monolocular cyst covered with a thin capsule, and pathological examination showed a thin-walled cyst lined by ciliated or nonciliated columnar cells resembling tubal epithelium. Immunohistochemical (IHC) analysis showed positive expression for paired box protein 8 (PAX8), Wilms' tumor 1 (WT-1), and estrogen receptors (ERs) and progesterone receptors (PRs). From the results of IHC analysis of control cases of mediastinal cystic tumors, four types of IHC analysis are quite helpful in obtaining a definitive diagnosis of Müllerian cyst. Although this cyst formation has been considered to be related to hormone imbalance, we suggest that hormone imbalance may be irrelevant to the etiology of Müllerian cyst, considering our case and two previous young cases. According to the fact that there is no significant difference between Müllerian cyst and non-Müllerian cyst in hematoxylin and eosin (HE) staining, a Mülleriancyst should always be considered in patients with a posterior mediastinal cyst, especially when it is adjacent to a thoracic vertebral body.
\end{abstract}

Keywords: Mediastinal Müllerian cysts; robot-assisted thoracoscopic surgery (RATS); immunohistochemical (IHC) staining; case report

Received: 06 June 2021; Accepted: 12 August 2021; Published: 30 March 2022.

doi: 10.21037/asj-21-46

View this article at: https://dx.doi.org/10.21037/asj-21-46

\section{Introduction}

Mediastinal cysts have a wide variety of origins; e.g., bronchogenic cysts, thymic cysts, or pericardial cysts (1); however, making a preoperative diagnosis of mediastinal cyst is usually difficult owing to the local anatomy. Hattori described the first case of a ciliated cyst of probable Müllerian origin arising in the posterior mediastinum (2), which is a very rare entity. Herein, we report a 25 -year-old female patient with a posterior mediastinal Müllerian cyst that was successfully removed by robot-assisted thoracoscopic surgery
(RATS). We present the following article in accordance with the CARE reporting checklist (available at https://asj. amegroups.com/article/view/10.21037/asj-21-46/rc).

\section{Case presentation}

The patient was a 25 -year-old woman who came to our hospital because of an incidental cyst in the posterior mediastinum. During the workup for her back pain, spinal magnetic resonance imaging (MRI) was performed,

^ ORCID: Yoshikane Yamauchi, 0000-0002-3374-2399; Yuichi Saito, 0000-0002-2025-9226; Yukinori Sakao, 0000-0002-6826-2280. 

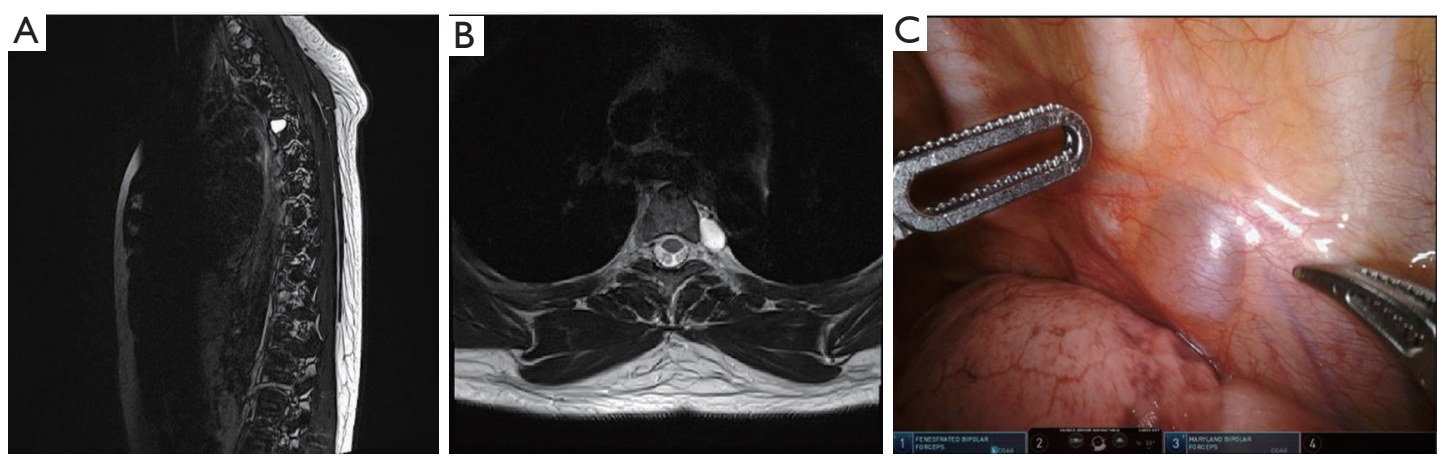

Figure 1 Magnetic resonance images. Sagittal (A) and axial (B) T2-weighted magnetic resonance images showing a hyperintense monolocular cystic lesion adjacent to the T4 vertebral body. (C) Intraoperative findings for this cystic lesion.

which revealed a well-circumscribed homogeneous mass, compatible with a simple cyst. The cyst measured $1.4 \mathrm{~cm} \times$ $1.0 \mathrm{~cm} \times 1.0 \mathrm{~cm}$ and was located along the left anterolateral side of the T4 vertebra (Figure 1). She was a healthyappearing, married woman, and a never-smoker and not obese (59.7 kg body weight; $157.7 \mathrm{~cm}$ tall). She had no family history of severe diseases, such as malignancies, and she was gravida 0 , para 0 . She was not taking medications or hormone therapy, and her back pain had already disappeared at her first visit to our hospital.

We suggested continuous monitoring of the cyst; however, the patient opted for its resection. RATS was performed for both diagnosis and treatment.

After the patient was placed in the left lateral decubitus position, the da Vinci Xi robot (Intuitive Surgical Inc., Sunnyvale, CA, USA), was docked. Intrathoracic $\mathrm{CO}_{2}$ insufflation and a 30-degree camera was used during the surgery. Four ports were installed at the sixth intercostal space, and Maryland bipolar forceps, fenestrated bipolar forceps, and Cadiere forceps were used. We located the cystic lesion at the T4 level adjacent to the left sympathetic trunk, intercostal veins, and intercostal nerves, and we were able to completely resect the lesion without rupture. The surgical time was 1 hour, console time was $24 \mathrm{~min}$, and a $20-F r e n c h$ chest tube was placed at the end of surgery. The chest tube was removed on the second postoperative day after confirming the absence of chylothorax. The patient was discharged on the third postoperative day, and her postoperative course was uneventful.

Pathological examination revealed that the lesion was covered with ciliated columnar epithelium, with underlying fibrous stroma. The epithelial cells were positive for estrogen receptors (ERs), progesterone receptors (PRs), paired box protein 8 (PAX8), and Wilms' tumor 1 (WT-1), by immunohistochemical (IHC) staining (Figure 2). The cyst was pathologically diagnosed as a mediastinal Müllerian cyst.

All procedures performed in this study were in accordance with the ethical standards of the institutional research committee and with the Helsinki Declaration (as revised in 2013). Written informed consent was obtained from the patient for publication of this case report and accompanying images. A copy of the written consent is available for review by the editorial office of this journal.

\section{Discussion}

The cyst wall was very thin and adjacent to important vessels and nerves; however, we completely resected the cyst without damage, thanks to the RATS approach. We consider that the RATS approach is helpful for thoracic surgeons when resecting Müllerian cysts, to avoid damaging the cyst wall. Furthermore, our patient was very satisfied with the fact that there was almost no postoperative wound pain, and that a reliable diagnosis was made with only small wounds. We also believe that it was easier to obtain an accurate diagnosis in this case because we avoided damaging the cyst wall; i.e., we did not damage the single layer of ciliated columnar epithelium. To the best of our knowledge, this is the third reported case of mediastinal Müllerian cyst in a woman less than 30 years old.

Most reported mediastinal Müllerian cysts have occurred in female patients during the perimenopausal period $(3,4)$; therefore, this cyst formation may be related to hormone imbalance. However, this does not apply in our case. Two cases of mediastinal Müllerian cysts in young women have been published $(2,5)$. Both cases were quite similar to our 

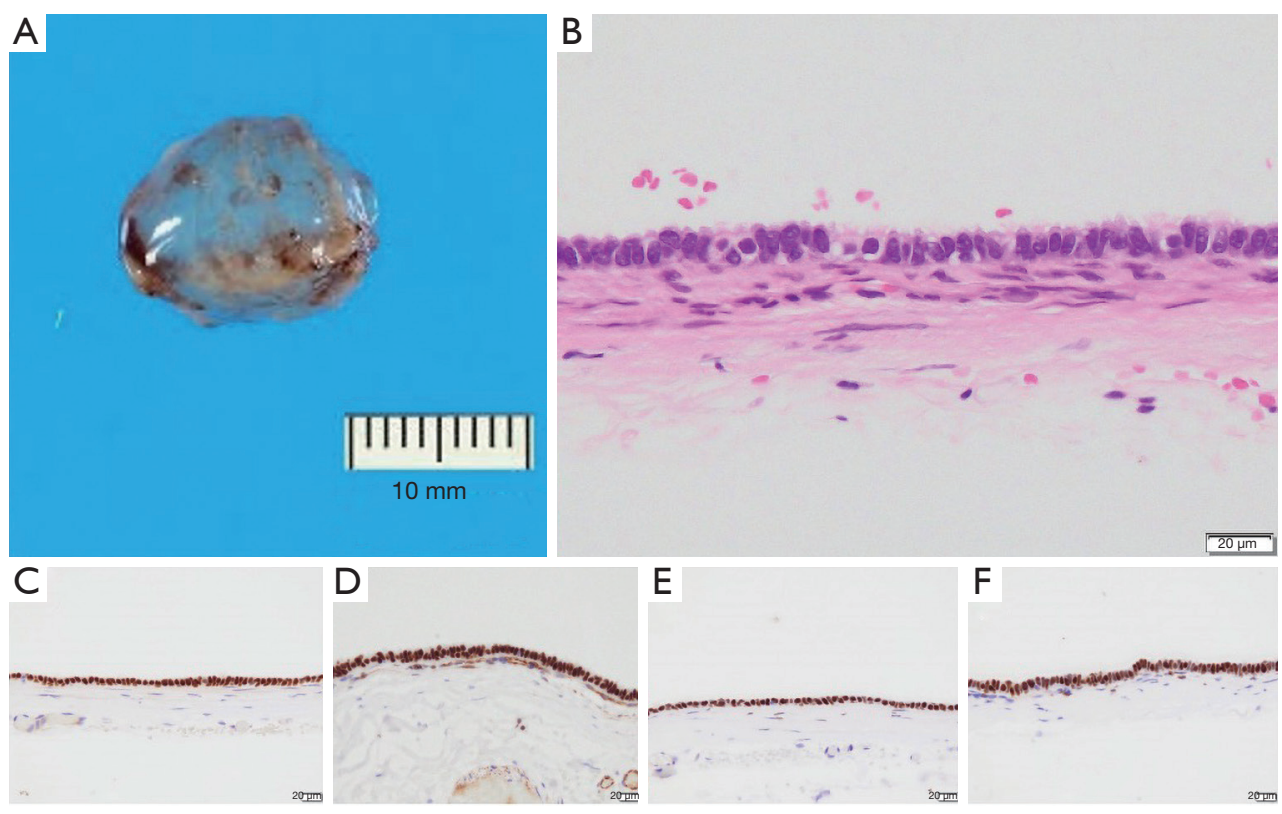

Figure 2 Pathological analysis. Macroscopic (A) and microscopic (B-F) appearance of the epithelium of the Müllerian cyst. The cyst wall was covered by a single layer of ciliated columnar epithelium (B). Ciliated columnar epithelium with positive immunoreactivity for PAX8 (C), WT-1 (D), ERs (E), and PRs (F) (B-F: bar: $20 \mu \mathrm{m}$, B, HE staining, C-F, IHC staining). PAX8, paired box protein 8; WT-1, Wilms' tumor 1; ERs, estrogen receptors; PRs, progesterone receptors. HE, hematoxylin and eosin; IHC, immunohistochemistry.

Table 1 Histopathologic comparison of Müllerian duct cyst, bronchogenic cyst, and thymic cyst

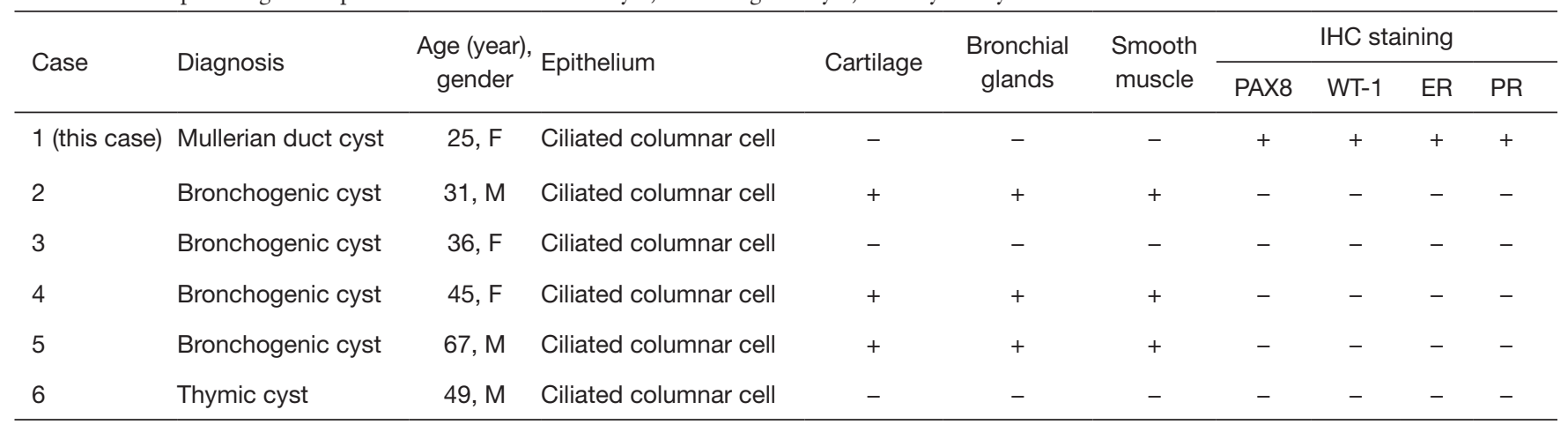

F, female; M, male; IHC, immunohistochemical; PAX8, paired box protein 8; WT-1, Wilms' tumor 1; ER, estrogen receptor; PR, progesterone receptor.

case regarding the incidental tumor detection and the lack of anatomical and hormonal abnormalities.

It is unknown how Müllerian duct cysts emerge in the posterior mediastinum, considering that these ducts originally develop in the retroperitoneum. Two theories have been proposed. Batt et al. suggested that the lesion derives directly from the residual tissue of the Müllerian apparatus at approximately stage 17 of embryogenesis (6). Hattori suggested that these cysts could represent misplaced mesothelium and mesenchyme with Müllerian characteristics (2). According to these theories, Müllerian cysts are congenital and not acquired.

From our case and the two previous young cases, we suggest that hormone imbalance may be irrelevant to the etiology of Müllerian cyst, considering that the incidence of hormonal therapy is higher in older women, and that the detection rate in annual checkups is also higher in older $v s$. younger women because older women are more likely to 
undergo annual checkups related to their age.

IHC staining is quite helpful in obtaining a definitive diagnosis of Müllerian cyst. Hattori reported the effectiveness of ER and PR expression in diagnosing 19 cases of mediastinal cysts, including three Müllerian cysts (7). Moreover, Simmons et al. confirmed PAX8 and WT-1 expression in Müllerian cysts, both of which are markers of Müllerian differentiation (8). We also examined ER, PR, PAX8, and WT-1 expression in five cases of mediastinal cystic tumors (four bronchogenic cysts and one thymic cyst) as controls (Table 1). In all cases, ER, PR, PAX8, and WT-1 expression was negative. These results indicate that this pattern of IHC staining is helpful in diagnosing Müllerian cyst. There is no significant difference between Müllerian cyst and non-Müllerian cyst in hematoxylin and eosin (HE) staining. Therefore, in accordance with our experience, we suggest that clinicians should consider Müllerian cyst as a possible diagnosis when the cyst is located in the posterior mediastinum, especially when adjacent to the thoracic vertebral body. Additionally, when Müllerian cyst is a possible diagnosis, IHC staining should be considered for a definitive diagnosis.

In conclusion, complete curative surgical removal of Müllerian cysts is the treatment of choice, as this approach establishes the diagnosis. Mediastinal Müllerian cyst should be included in the differential diagnosis of posterior mediastinal cysts.

\section{Acknowledgments}

We thank Jane Charbonneau, DVM, from Edanz Group (https://jp.edanz.com/ac) for editing a draft of this manuscript.

Funding: None.

\section{Footnote}

Reporting Checklist: The authors have completed the CARE reporting checklist. Available at https://asj.amegroups.com/ article/view/10.21037/asj-21-46/rc

Peer Review File: Available at https://asj.amegroups.com/ article/view/10.21037/asj-21-46/prf

Conflicts of Interest: All authors have completed the ICMJE uniform disclosure form (available at https://asj.amegroups. com/article/view/10.21037/asj-21-46/coif). The authors have no conflicts of interest to declare.

Ethical Statement: The authors are accountable for all aspects of the work in ensuring that questions related to the accuracy or integrity of any part of the work are appropriately investigated and resolved. All procedures performed in this study were in accordance with the ethical standards of the institutional research committee and with the Helsinki Declaration (as revised in 2013). Written informed consent was obtained from the patient for publication of this case report and accompanying images. A copy of the written consent is available for review by the editorial office of this journal.

Open Access Statement: This is an Open Access article distributed in accordance with the Creative Commons Attribution-NonCommercial-NoDerivs 4.0 International License (CC BY-NC-ND 4.0), which permits the noncommercial replication and distribution of the article with the strict proviso that no changes or edits are made and the original work is properly cited (including links to both the formal publication through the relevant DOI and the license). See: https://creativecommons.org/ licenses/by-nc-nd/4.0/.

\section{References}

1. Takeda S, Miyoshi S, Minami M, et al. Clinical spectrum of mediastinal cysts. Chest 2003;124:125-32.

2. Hattori H. Ciliated cyst of probable mullerian origin arising in the posterior mediastinum. Virchows Arch 2005;446:82-4.

3. Saad Abdalla Al-Zawi A, Idaewor P, Asaad A, et al. Posterior Mediastinal Paravertebral Müllerian cyst (cyst of Hattori): literature review. Adv Respir Med 2020;88:134-41.

4. Thomas-de-Montpréville V, Dulmet E. Cysts of the posterior mediastinum showing müllerian differentiation (Hattori's cysts). Ann Diagn Pathol 2007;11:417-20.

5. Lee JE, Cha YK, Kim JS, et al. Müllerian Cyst in Posterior Mediastinum in a Young Woman. Balkan Med J 2018;35:216-8.

6. Batt RE, Mhawech-Fauceglia P, Odunsi K, et al. Pathogenesis of mediastinal paravertebral müllerian cysts of Hattori: developmental endosalpingiosis-müllerianosis. Int J Gynecol Pathol 2010;29:546-51.

7. Hattori H. High prevalence of estrogen and progesterone 
receptor expression in mediastinal cysts situated in the posterior mediastinum. Chest 2005;128:3388-90.

8. Simmons M, Duckworth LV, Scherer K, et al. Mullerian cysts of the posterior mediastinum: report of two cases and review of the literature. J Thorac Dis 2013;5:E8-E10.

doi: 10.21037/asj-21-46

Cite this article as: Yamauchi Y, Saito Y, Saito K, Asami M, Haga T, Sasajima Y, Kanamoto Y, Yokote F, Dejima H, Sakao Y, Kawamura M. A case report: robot-assisted resection of a mediastinal Müllerian cyst in a young woman. AME Surg J 2022;2:10. 